\title{
COMPENSATION OF FRICTION IN ROBOTIC ARMS AND SLIDE TABLES
}

\author{
Tomasz Żabiński*, Andrzej Turnau** \\ * Computer and Control Engineering Chair, Rzeszów University of Technology \\ W. Pola 2, 35-959 Rzeszów, Poland, \\ e-mail: tomz@prz-rzeszow.pl \\ **Institute of Automatics, University of Science and Technology \\ Mickiewicza 30, 30-059 Kraków, Poland \\ e-mail: atu@ia.agh.edu.pl
}

\begin{abstract}
The paper presents experimental results of friction parameter identification for two different systems: rotational arms of a direct-drive spherical manipulator and linear slide tables. Parameters of the static part of the LuGre friction model are estimated. Several experiments are reported in which selected friction compensation methods are employed for tracking at low velocities. Two compensators working in real time are constructed and verified: the PD-like lead, to avoid stick-slip motion and the on-line trained neural network with a special structure, to avoid errors caused by friction and varying dynamics of robotic actuators. Copyright $\odot 2005$ IFAC
\end{abstract}

Keywords: Direct-Drive Robots, Friction, Compensation, PD-Controllers, Neural control, Positioning systems

\section{INTRODUCTION}

Friction is a highly nonlinear phenomenon found in all mechanical systems. From the point of view of control, friction causes the following unfavorable effects: stick-slip motion, significant tracking errors, large settling time and limit cycles. Control methods used for precise and high-performance motion systems and also complex control algorithm (like optimal control) require efficient compensation of friction. Theoretically a rule compensating friction is simple: apply a force opposite to the instantaneous friction force. However, it is difficult to guarantee the robustness in both stability and performance criteria when fixed model friction compensation is used because friction is nonstationary and positiondependent phenomena. Thus methods that do not require a complete knowledge of the friction dynamics (Tataryn, et al. 1996) have significant practical meaning. Many of such methods have been developed for example: stiff PD, dither, impulsive control, smooth robust nonlinear feedback, etc. At the same time many intensive studies are being worked on to develop and identify friction models.
Among those friction models already described in different sources the most popular and mainly accepted one is the LuGre (Canudas, et al. 1995) model with its modifications. The LuGre model links both steady-state friction curve and friction dynamic characteristic. This model, although relatively simple, reflects most of friction induced phenomena very significant for feedback control. Unfortunately, a good estimation of model parameters (especially parameters of dynamic part of the model) is often quite difficult to be achieved in practice, especially when typical industrial control environment and sensors are used. An excellent review of models, analysis and control tools for friction compensation can be found in the survey paper by ArmstrongHélouvry, et al. (1994).

In this paper the experimental results of friction parameter identification for two different systems: rotational arms of a direct-drive spherical robot and linear slide tables are discussed. To describe nonlinear friction torques occurring in the systems the LuGre model focused on the steady-state friction curve is used. The main part is devoted to friction compensation methods. A lead compensator for 
tracking at low velocity (Armstrong-Hélouvry, et al. 1994) is employed to avoid stick-slip motion. Other compensation technique is based on neural networks $\mathrm{NN}$. There are the NN of special type (Lewis, et al. 2002). The NN weights are tuned in such a way that NN learns about the friction nonlinearity on line.

A PC-based real-time architecture (RT-CON, 1998) is used to collect the data necessary to identify friction model parameters and to perform control experiments with friction compensation.

\section{FRICTION MODELLING}

There are static and dynamic friction models. Static models include the observed friction phenomena like: Coulomb, viscous, static friction and Stribeck effect and their possible combinations. They do not include frictional memory. Dynamic friction models are more complex. They describe such phenomena as: presliding displacement, or frictional lag. A number of the dynamic models are proposed: Dahl, Bliman, Sorine, LuGre and others. An excellent and brief review of models can be found in the Ph.D. project by Hensen (2002).

The LuGre model corresponds to: steady-state friction curve and the presliding phase by means of flexible bristles, representing the contact points of the moving surfaces. The basic model has the form (Canudas, 2003)

$$
\begin{gathered}
\frac{d z}{d t}=v-\frac{\sigma_{0}}{g(v)} z|v| \\
F=\sigma_{0} z+\sigma_{1} \frac{d z}{d t}+f(v)
\end{gathered}
$$

where $z$ denotes the average bristle deflection. The model behaves like a spring for small displacement where $\sigma_{0}$ is the stiffness of the bristles and $\sigma_{l}$ the damping of the elastic bristles. The function $g(v)$ describes steady-state friction curve and $f(v)$ is the viscous friction. An equation of $g(v)$ that has been proposed (Canudas, et al. 1995) to describe the Stribeck effect is

$$
g(v)=F_{c}+\left(F_{s}-F_{c}\right) e^{-\left(v / v_{s}\right)^{2}}
$$

where $F c$ is the Coulomb friction, $F s$ is the static friction force and $v_{s}$ is the Stribeck velocity. A modified description of the Stribeck curve has been proposed by Bona et al. (2003) in the form of

$$
g(v)=\alpha_{0}+\alpha_{1} e^{-\left(v / v_{1}\right) \operatorname{sgn}(v)}+\alpha_{2}\left(1-e^{-\left(v / v_{2}\right) \operatorname{sgn}(v)}\right)
$$

which gives possibility to achieve better data fitting $\left(F_{s}=\alpha_{0}+\alpha_{1}, \quad F_{c}=\alpha_{0}+\alpha_{2}\right)$. The steady-state part of the LuGre model (Canudas, et al. 1995)

$$
F_{s s}=g(v) \operatorname{sgn}(v)+f(v)
$$

is used here to describe nonlinear friction torques. Viscous friction can be described as a linear function of velocity (Canudas, et al. 1995)

$$
f(v)=F_{v} v
$$

or like Bona et al. (2003) for direct-drive systems

$$
f(v)=F_{v} v+F_{v 2} v^{2}
$$

\section{THE EXPERIMENTAL SETUP}

The experimental setup consists of two different mechanical systems: 3DOF robot and two linear slide tables with mechanical components of different types. The considered robot (see Fig. 1) is a spherical three-link laboratory manipulator. Two rotate links are driven by brush-less NSK Megatorque (MYS3040GG001) direct-drives motors with ESA25 drivers (M-ESA-Y3040T25). The prismatic link is driven by linear motor and will be investigated further in the future.

ESA25 drivers have three basic control modes: torque, velocity and position. As a position sensor a resolver with 38400 pulses per revolution is used. The sample time for all control loops in the driver is equal to $555 \mu \mathrm{s}$. The torque mode is defined for all experiments.

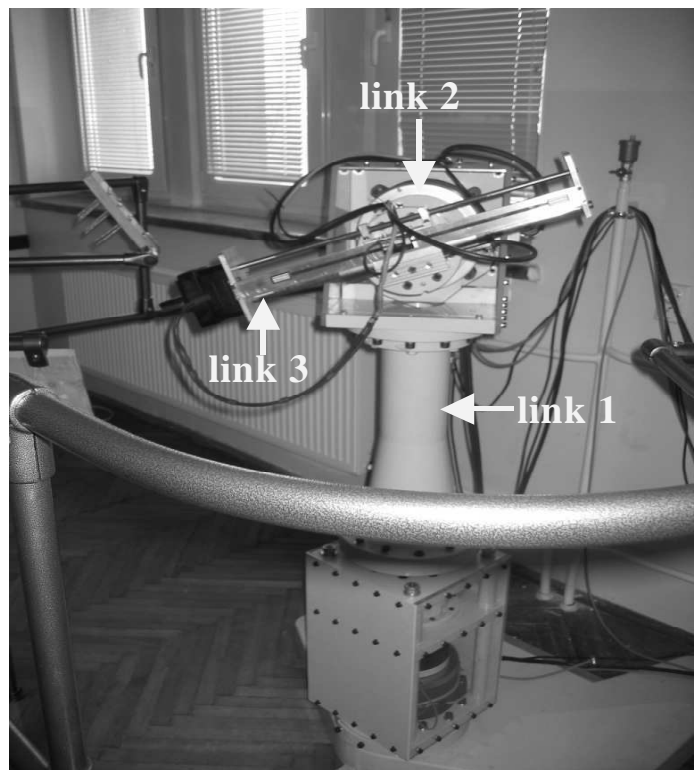

Fig. 1. Experimental setup - 3DOF robot.

The first linear slide table consists of Thomson Superslide System which is a ball screw (length 38 $\mathrm{cm}$, diameter: $12 \mathrm{~mm}$ ) actuated system with linear ball guides. The second system consists of DCI linear component with lead screw (length $30 \mathrm{~cm}$, diameter: $15 \mathrm{~mm}$ ) and linear slide guides. Both systems are equipped with DC motors (no gears) with amplifiers working in current mode and optical encoders with 2048 pulses per revolution for position measurements. The Thomson system unlike DCI consists of mechanical elements developed to minimize friction. The systems are only equipped with position sensors so velocity signals are estimated using position measurements.

The control architecture consists of a PC computer equipped with Windows XP operating system and 
PCI input/output board - RT-DAC4 (RT-DAC, 1997). Real-time control is achieved by using RTCON (RT-CON, 1998) toolbox which interacts with Matlab/Simulink environment. Due to RT-CON one may perform control, capture and analyze data and change "on-the-fly" parameters of the running realtime control system.

\section{DYNAMICS AND FRICTION MODEL IDENTIFICATION}

The model of the manipulator under study can be described by the following equation

$$
M(q) \ddot{q}+C(q, \dot{q}) \dot{q}+\tau_{f}(q, \dot{q})=\tau
$$

where $q, \dot{q}$ and $\ddot{q}$ are the vectors of joint angels, angular velocities and angular accelerations, $M$ is the configuration dependent inertia matrix, $C$ is the term containing Coriolis torques, $\tau_{f}$ is the friction torque vector and $\tau$ is the command torque vector. For the presented study the only two rotating links are taken into account. Under these conditions the gravitational effects are considered to be negligible and the manipulator links will be considered as independent simple rotating links. Due to these assumptions the single link can be modeled as

$$
J \ddot{q}=\tau-\tau_{f}(q, \dot{q})
$$

where $J$ is the effective inertia, $q$ angular position, $\tau$ motor torque and $\tau_{f}$ is the friction torque. The motor with driver is assumed to be an ideal torque source so $\tau$ can be described as

$$
\tau=k_{v 2 \tau} u
$$

where $u$ is the input voltage and $k_{v 2 \tau}$ is the voltage to torque gain. This is a common assumption in literature (Bona et al. 2003). The similar equation as (8) describes linear slide tables. However, unlike in the robot system, it is possible to measure current not only input voltage $u$ so

$$
\tau=k_{i 2 \tau} i
$$

where $i$ is the input current, $k_{i 2 \tau}$ is the current to torque gain. For the NSK motors $k_{v 2 \tau}$ equals to 4 $\mathrm{Nm} / \mathrm{V}$. For the linear slide tables and Thomson - DCI $k_{i 2 \tau}$ equals respectively to $0.073 \mathrm{Nm} / \mathrm{A}$ and $0.2 \mathrm{Nm} / \mathrm{A}$. Identification of equivalent inertia $J$ and viscous friction is performed according to procedure proposed by Canudas (2003) under an assumption of large velocities of motion. In the motionless system we can estimate the static friction if only it starts to rotate. Obtained results are presented in Table 1 (a symmetric model is assumed).

For identification of a steady-state friction curve in the robot links three different types of experiments are performed. The first one is an open loop experiment in which the motor rotates freely until a dynamic equilibrium situation at constant velocity is achieved. The second experiment drives a system with constant velocity using a PD control law or a lead compensator to avoid the stick-slip phenomena.

Table 1 Experimental results of inertia, viscous and static friction estimation

\begin{tabular}{|c|c|c|c|}
\hline & $J\left[\mathrm{kgm}^{2}\right]$ & $F_{s}[\mathrm{Nm}]$ & $F_{v}[\mathrm{Nms}]$ \\
\hline Link 1 & 0.57 & 4.4 & 1.92 \\
\hline Link 2 & 0.038 & 2.4 & 0.55 \\
\hline Thomson & 0.000012 & 0.03 & 0.00011 \\
\hline DCI & 0.000047 & 0.025 & 0.00015 \\
\hline
\end{tabular}

The third experiment drives the system with a constant acceleration and the friction force is obtained from the equation

$$
\tau_{f}(\dot{q})=\tau-J \ddot{q} .
$$

The first experiment gives valid results for high velocities and the second for low velocities. Due to the system dynamics the measurements obtained for the high velocities in the second type test do not agree with the results from the first type experiments. The third type test, due to the necessity to calculate the second derivative of position measurements, needs an extensive filtering and is used only for verification of the results obtained from the first and the second type of experiments. Figure 2-5 and Tables $2 \& 3$ show results of identification.
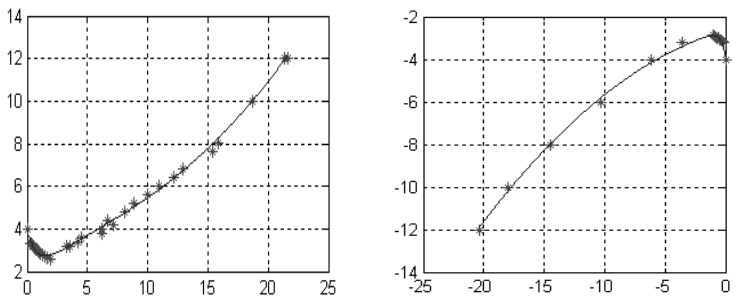

Fig. 2. Friction torque (Nm) vs. positive and negative velocity $(\mathrm{rad} / \mathrm{s})$ on Link 1 .
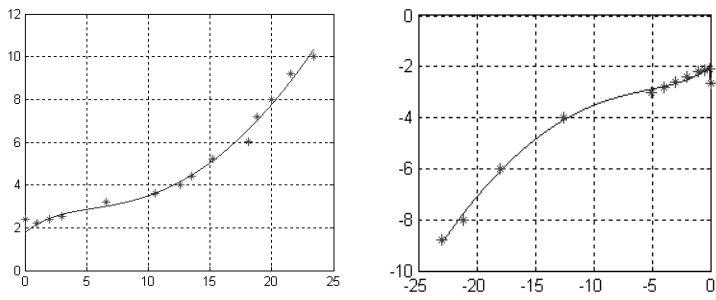

Fig. 3. Friction torque (Nm) vs. positive and negative velocity $(\mathrm{rad} / \mathrm{s})$ on Link 2.
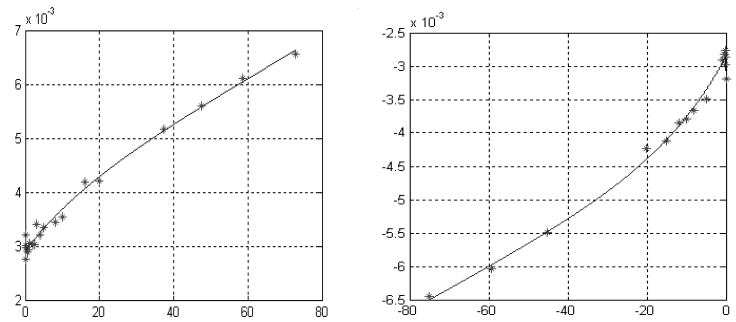

Fig. 4. Friction torque (Nm) vs. positive and negative velocity $(\mathrm{rad} / \mathrm{s})$ on Thomson system. 

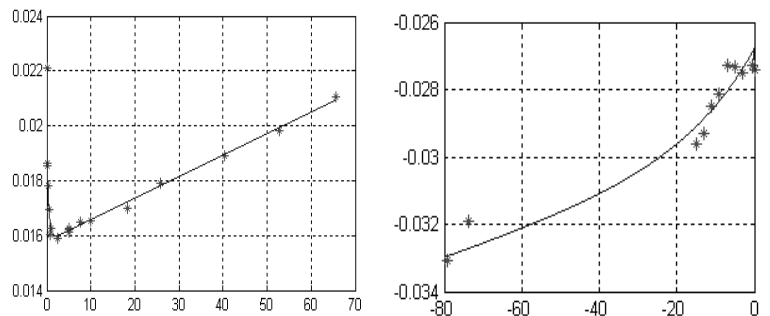

Fig. 5. Friction torque $(\mathrm{Nm})$ vs. positive and negative velocity $(\mathrm{rad} / \mathrm{s})$ on DCI system.

Values from Table 1 become a starting point for least-squares algorithm used for fitting the steadystate friction curve.

The use of expression 6 to describe viscous friction in manipulator system is motivated by achieving a better data fitting like in Bona et al. (2003) for the similar system. For the linear slide table systems the expression 5 describes accurately behaviors of the systems.

One can notice that the curves shown in Figures 2-5 have different shapes but reflect similar phenomena like growing friction force for higher velocities and the Stribeck effect for low velocities.

The results agree with shapes of friction curves presented by Pritschow (1995) for systems with linear ball guides (Fig. 4) and linear guides without extensive lubrication (Fig. 5). Very similar mechanical systems such as Link 1 and Link 2 differ significantly (Fig. 2 and 3) in the velocity range and the level of the Stribeck effect. The Stribeck effect is mostly detectable in Link 1 where a larger amount of grease has been used. The influence of this effect is very important in the system. The observed influence of the amount of grease on the Stribeck effect agrees with the charts presented by Pritschow (1995).

Regardless of shapes of the steady-state friction curves the stick-slip motion is observed in all presented systems.

The identification procedure used here is timeconsuming and in the future the frequency domain identification method proposed by Hensen (2002) will be used. Current work is devoted to achieve better fitting of the steady-state friction curves especially for friction on low velocities. The obtained friction curves are treated as an average friction characteristic due to the nonstationary and positiondependent friction feature, especially for linear slide tables.

Table 2 Estimated parameters of the steady-state friction curves described by equations 3 and 4 and 6 for the manipulator

\begin{tabular}{|c|c|c|c|c|}
\hline & $\begin{array}{c}\text { Link 1 } \\
v>0\end{array}$ & $\begin{array}{c}\text { Link 1 } \\
V<0\end{array}$ & $\begin{array}{c}\text { Link 2 } \\
v>0\end{array}$ & $\begin{array}{c}\text { Link 2 } \\
v<0\end{array}$ \\
\hline$\alpha_{0}$ & 1.4396 & 2.6364 & 1.7373 & 1.9904 \\
\hline$\alpha_{1}$ & 2.4848 & 1.2772 & 0.6627 & 0.6497 \\
\hline$v_{1}$ & 0.8962 & 0.3535 & 0.0001 & 0.0001 \\
\hline$\alpha_{2}$ & 2.8494 & 0.8615 & 2.9713 & 2.3236 \\
\hline$v_{2}$ & 4.1190 & 18.6965 & 3.3923 & 3.7337 \\
\hline$F_{v}$ & -0.0483 & 0.1084 & -0.3653 & -0.2684 \\
\hline$F_{v 2}$ & 0.0190 & 0.0156 & 0.0259 & 0.0204 \\
\hline
\end{tabular}

Table 3 Estimated parameters of steady-state friction curves described by equations 3 and 4 and 5 for linear slide tables

\begin{tabular}{|c|c|c|c|c|}
\hline & $\begin{array}{c}\text { Thomson } \\
v>0\end{array}$ & $\begin{array}{c}\text { Thomson } \\
v<0\end{array}$ & $\begin{array}{c}\text { DCI } \\
v>0\end{array}$ & $\begin{array}{c}\text { DCI } \\
v<0\end{array}$ \\
\hline$\alpha_{0}$ & 0.0028 & 0.0028 & 0.019 & 0.0267 \\
\hline$\alpha_{1}$ & 0.0003 & 0.00034 & 0.0029 & 0.0006 \\
\hline$v_{1}$ & 0.0001 & 0.0001 & 0.0001 & 0.0001 \\
\hline$\alpha_{2}$ & 0.00082 & 0.0012 & -0.003 & 0.0031 \\
\hline$v_{2}$ & 15.99 & 15.9 & 0.436 & 18.89 \\
\hline$F_{v}$ & 0.00004 & 0.00003 & 0.00007 & 0.000039 \\
\hline
\end{tabular}

The results presented in Tables 2 and 3 are used to compensate stick-slip friction in the systems as shown in the next paragraph.

\section{STICK-SLIP MOTION PD COMPENSATION}

A stick-slip motion is present in the considered systems. Figs. 6 and 7 show the examples of the stick-slip motion for Link 1 and the Thomson system respectively.

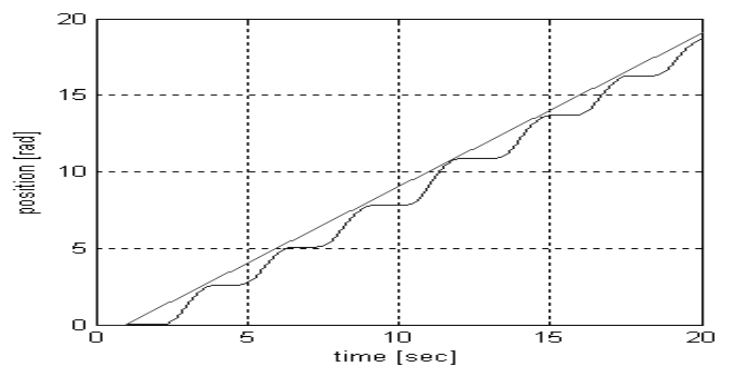

Fig. 6. Stick-Slip motion in Link 1 for $1 \mathrm{rad} / \mathrm{sec}$ velocity.

Several methods of the stick-slip compensation are tested. The main element of a controller is a simple PD control. The model-based compensation methods concerned with obtained steady-state friction curves like feed-forward (the desired velocity is used) or feedback control (the measured velocity is used) give a slight improvements on system performance. It proves that identified friction parameters represent friction in the mean sense. Therefore a further work is needed and the full dynamic model with adaptation or observers should be used for compensation.

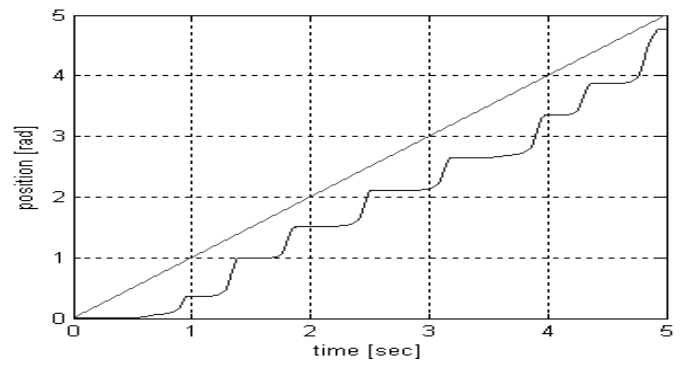

Fig. 7. Stick-Slip motion in the Thomson system for $1 \mathrm{rad} / \mathrm{sec}$ velocity.

A simple stiff PD control (without modeling) is applied and due to Tataryn, et al. (1996) a smooth 
robust nonlinear feedback (SRNF) is tested. The usage of a simple stiff PD control in the systems is limited because of its sensitivity to noise generated by the digital derivative of position measurements. However, for Link 2 stiff PD compensation method for tracking system with velocities growing from $0.01 \mathrm{rad} / \mathrm{sec}$ is sufficient to avoid the stick-slip motion. For Link 1 (due to the Stribeck effect) and for linear slide tables (due to the vibrations produced by flexibility caused - among others - by Helical couplings) the stiff PD control is not sufficient to the same control purpose. Investigated by Tataryn, et al. (1996) the smooth robust nonlinear feedback compensation method works well for the entire system except the Link 1 . For Link 1 it is not possible to set up parameters of SRNF to eliminate stick-slip (like shown in Figure 8).

Because of problems with eliminating stick-slip motion for Link 1, the other form of PD control law is used (PD like lead compensator controller (Trybus, 2003)). A proportional-plus-derivative (PD) control is standard for tracking loops in industrial systems. The compensator is described by the following equation

$$
\mathrm{PD}=k_{\mathrm{p}}\left(1+\frac{T_{\mathrm{d}} s}{\frac{T_{\mathrm{d}}}{\mathrm{D}} s+1}\right)=k \frac{s+z}{s+p}
$$

where $k, z$ and $p$ are chosen during a design process. The compensator parameters are calculated using the root locus design method (settling time and overshoot as an input data) with the assumption that actuator could be described as a current driven motor with the viscous friction. The calculated compensator parameters corresponding to data from Table 1 are given in Table 4.

Table 4 Examples of PD compensator parameters

\begin{tabular}{|c|c|c|c|}
\hline & $\mathrm{k}$ & $\mathrm{z}$ & $\mathrm{p}$ \\
\hline Link 1 & 6.4 & 2 & 8 \\
\hline Link 2 & 19.68 & 16 & 64 \\
\hline Thomson & 0.64 & 40 & 160 \\
\hline DCI & 2.73 & 80 & 320 \\
\hline
\end{tabular}

The compensator unlike SRNF provides the smooth motion (see Fig. 8). It eliminates the stick-slip motion in the robot including Link 1. The compensator provides smooth motion but with a steady-state tracking error. To eliminate this error the neural network is proposed in section 7 .

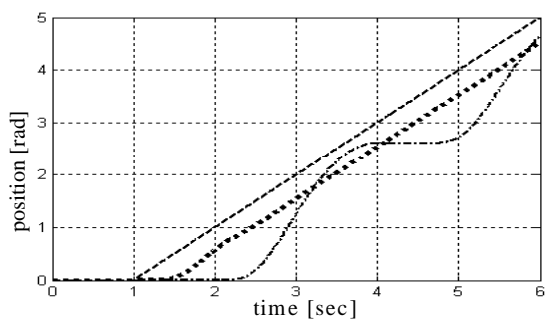

Fig. 8. Stick-Slip compensation in Link 1 for 1 $\mathrm{rad} / \mathrm{sec}$ velocity with SRNF compensation (dash-dot) and PD like lead compensator (dot).

\section{ON-LINE NEURO-COMPENSATION OF FRICTION AND DYNAMICS OF ROBOT LINK}

Two neural networks: standard (NN) and augmented (ANN) are used as compensators. This method has been proposed and solved by simulation in Lewis, et al. (2002). Both networks consist of two layers. NN has two neurons in the hidden layer and one neuron in the output layer. The input vector consists of: position error, its derivative, reference position, its derivative and its second derivative. There is twodimensional sigmoid activation vector in hidden layer and one linear activation function in the output neuron. As far as ANN is concerned one neuron with a piecewise continuous activation function is added in the hidden layer. This neuron has only one input activated by the velocity signal. Its weight and threshold are selected to be fixed to correspond to the known point of friction discontinuity (at velocity 0 ). During motion the weights of both layers except this one mentioned above are permanently trained by modified backpropagation method (Lewis, et al. 2002) and updated on-line (in each control sample).

Experiment 1 of real-time motion tracking of link 2 with fixed velocity is given in Figures 9 and 10.

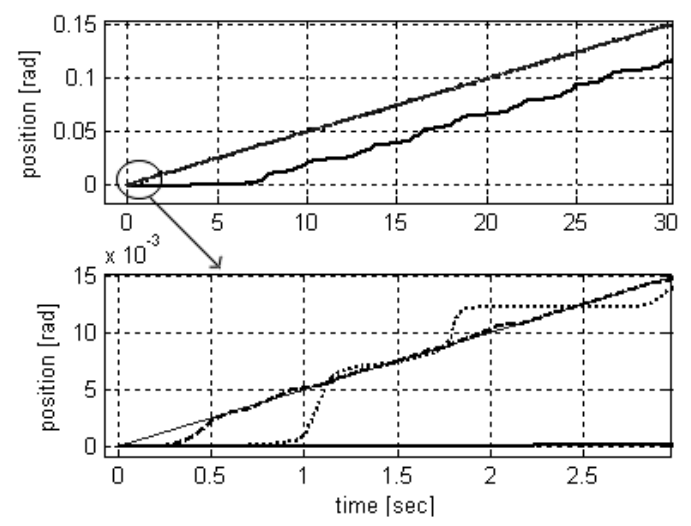

Fig. 9. Angular position of link 2 with friction compensators: NN (dot), ANN (dash) and PD (bold).

Constant velocity $0.005 \mathrm{rad} / \mathrm{s}$ is applied.

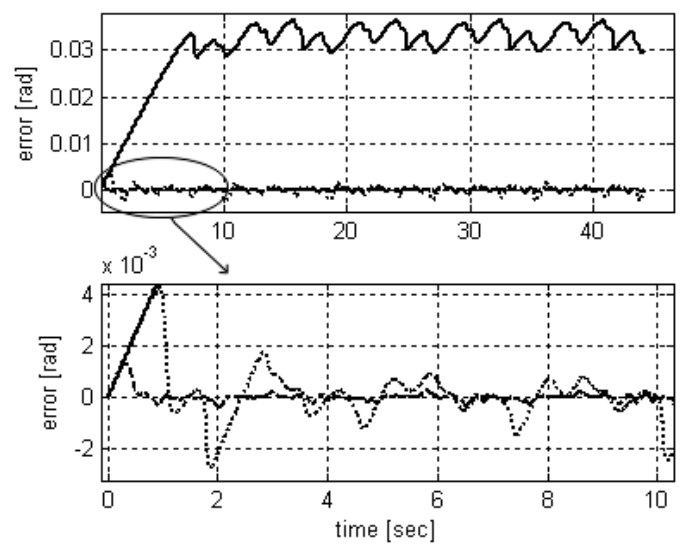

Fig. 10. Tracking errors of link 2 with friction compensators: NN (dot), ANN (dash) and PD (bold).

Constant velocity $0.005 \mathrm{rad} / \mathrm{s}$ is applied. 
Experiment 2 besides the angular motion of link 2 introduces the straight-line motion of link 3. A sinusoidal profile of this motion is shown in Figure 11 (upper diagram). The link 2 rotates with constant velocity $0.005 \mathrm{rad} / \mathrm{s}$ being disturbed (due to gravitational forces) by motion of link 3. Link 3 starts from the horizontal position.
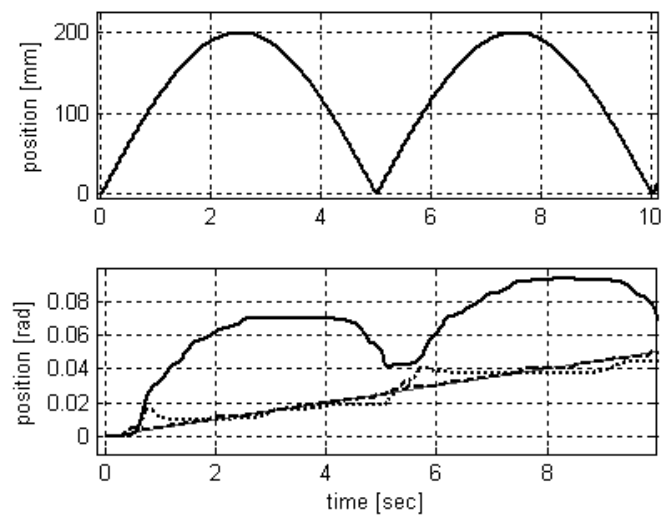

Fig. 11. Upper diagram - gripper position in $\mathrm{mm}$. Lower diagram - angle of link 2 with compensators: NN (dot), ANN (dash), PD (bold).

At the beginning the hidden layer weights are defined as random values and the output layer weights are set to zero. All weights in the hidden and output layers are updated except the weight being input to augmented neuron.

The result of training shows that hidden weights are only slightly modified during continuous process of learning. On the contrary the weights of output layer are changing significantly. The weights of NN are updated to follow periodical changes in the dynamics of link 3 and also varying frictions in the robot joints. The changing weights are shown in Fig. 12. They correspond to Experiment 2 (see Fig. 11). The PD compensator is not sufficient to reduce effects of varying dynamics. NN learned on line is competitive to a simple PD. Moreover ANN outperforms common NN and the angular motion of link 2 remains linear in the whole motion range of link 3 .
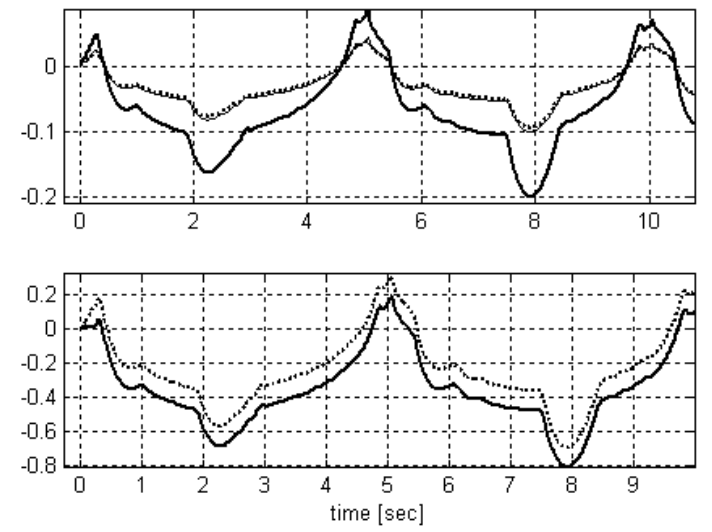

Fig. 12. On-line learning of neural network weights. Upper diagram - output layer: First weight (solid), Second weight (dot), bias (bold). Lower diagram output layer: the augmented neuron weight (bold), bias (dot).

\section{CONCLUSIONS}

Usually we have to deal with friction that cannot be reduced. The PD-like lead, NN and Augmented NN compensators are introduced to get rid of friction and varying dynamics effects. These compensators are designed and applied in the real time robotic systems. In this way we have proved by experiments that the simulated NN and ANN controllers - proposed by Lewis, et al. (2002) operate successfully in real-time world. This is an important step toward applications of complex control algorithms.

\section{ACKNOWLEDGEMENTS}

The work has been partially supported by Polish State Committee for Research - research grant 3T11A 01227.

\section{REFERENCES}

Armstrong-Hélouvry, Dupont B., P. and Canudas de Wit C. (1994). A survey of models, analysis tools and compensation methods for the control of machines with friction. Automatica, 30, 1083-1138.

Bona B., Indri M., Smaldone N. (2003). Nonlinear friction estimation for digital control of directdrive manipulators. In European Control Conference, Cambridge.

Canudas de Wit C., Olsson H., Åström K. J., Lischinsky P. (1995). A New Model for Control of Systems with Friction. IEEE Trans. on Automatic Control, 40 (3), 419-425.

Canudas de Wit, C. (2003). Modeling and Control of Systems with Dynamic Friction. MiniCourse on: Control of Systems with Dynamic Friction.

Hensen, Ronnie H.A. (2002). Controlled Mechanical Systems with Friction. Ph.D. thesis Eindhoven University of Technology, Department of Mechanical Engineering, Systems and Control Group.

Lewis, F. L., Campos J., Selmic R. (2002). NeuroFuzzy control of industrial systems with actuator nonlinearities. SIAM.

Pritschow G. (1995). Control techniques of lathes and industrial robots. Wrocław (in Polish).

RT-DAC, (1997). RT-DAC multi I/O board, Users Manual, InTeCo Ltd; www.inteco.com.pl .

RT-CON, (1998). RT-CON Professional, Users Manual, InTeCo Ltd; www.inteco.com.pl.

Tataryn, P.D., Sepehri N. and Strong D. (1996). Experimental comparison of some techniques for the control of manipulators with stick-slip friction. Control Eng. Practice, 4 (9), 12091219.

Trybus, L. (2003). Feedback Control - lecture. Rzeszów University of Technology, Computer and Control Engineering Chair. 